\title{
Impacto en los ingresos de la población de Tegucigalpa debido a estudios superiores, año 2012
}

Héctor Enrique Moncada ${ }^{1}$

Erwin Arrone ${ }^{2}$

\section{RESUMEN}

La educación ejerce un impacto sobre el aumento del ingreso de los individuos, medido a través de la tasa de rendimiento educativo. El estudio fue desarrollado en la ciudad universitaria tomando en cuenta las ramas de la actividad económica. La base de los años de escolaridad para determinar los niveles de la educación superior; considerando el primero al nivel de licenciatura y el segundo a nivel de maestría.

El rendimiento de la educación sobre los ingresos juega un rol importante en el aumento del valor monetario recibido por los individuos y en el diseño de políticas públicas salariales del Gobierno y mercado laboral.

En los resultados obtenidos de la investigación, los rendimientos educativos proveen mejor nivel de ingresos a los hombres que a las mujeres, a esto se le agrega que los hombres que laboral en la industria manufacturera tienen mejor probabilidad de aumentar sus ingresos que la mujer.

Según el nivel educativo, los individuos que ostentan el nivel de maestría obtienen una mayor tasa de retorno en los ingresos que el de licenciatura; sin embargo, entre regiones geográficas la ciudad de San Pedro Sula obtuvo mayor retorno que en el Distrito central en el nivel de maestría. Conclusiones como las anteriores se destacan en esta investigación mediante la estimación de un modelo transversal, semilogarítmico de retornos marginales a la educación superior.

Palabras clave: retornos marginales, años de escolaridad, tasa de retorno, nivel de educación de licenciatura, nivel de educación de maestría, experiencia.

\footnotetext{
${ }^{1}$ Beneficiario de una beca de estudiante de posgrado de la DICYP, Maestría en Metodologías de la Investigación Económica y Social, UNAH: mhectorenrique@yahoo.es

${ }^{2}$ Asesor Metodológico, profesor del departamento de Economía, Facultad de Ciencias Económicas, UNAH.
} 


\section{ABSTRACT}

Analyzing whether education has an impact on increasing revenues, this measure through the rate of educational achievement, this article is built with information I get from the University, having two large geographical areas, and two industries. Based on years of schooling to higher levels of education; level of university degree and master's degree. This operation for education has an important role in improving the income of individuals, which plays an important role in the design of wage policies of the government and the labor market. However, the yields offered a better educational level of earnings among men than women. In addition, men who work in the manufacturing industry have a better chance of increasing their income than women. According to the level of education, the teacher gets more revenue than the bachelor. However in San Pedro Sula, university graduates were better paid than those who obtained their master's degree. This research was presented through the estimation of a transversal model, semi-logarithmic marginal returns to higher education.

Keywords: marginal returns, higher education years, rate of return, undergraduate level, master's level, experience. 


\section{INTRODUCCIÓN}

La premisa de que los individuos desean alcanzar mayores niveles de ingresos que les permitan mejorar su condición de vida a través de la inversión en educación, los lleva a incrementar los niveles de escolaridad. De esta manera, las personas necesitan mejorar sus ingresos y, por ende, su calidad de vida y la de sus familias, lo cual logran a través del aumento de sus niveles de escolaridad para obtener un trabajo digno y bien remunerado, es decir, se busca, una relación de causalidad dada por el variable ingreso y años de escolaridad.

Según el Informe de Desarrollo Humano elaborado por el programa de las Naciones Unidas para el desarrollo (PNUD, 2011) mencionan que el mercado pre $\urcorner$ mia a quienes tienen educación, lo que constituye un estímulo para que las familias y las personas se interesen en que sus miembros aprovechen la oferta educacional. Sin embargo, la población nacional mantiene un promedio de años de educación de 6.4 y esto se ahonda el promedio nacional del ingreso mensual de las poblaciones del Distrito Central y San Pedro Sula es de L. 5, 337.00 (INE, 2011); indicadores importantes al analizar el aumento de la escolaridad y su retribución en el ingreso y así comprobar el beneficio de aumentar los años de escolaridad.

Analizando el aumento en años de escolaridad y el impacto sobre los ingresos mensuales de los individuos residentes en Tegucigalpa como objetivo primordial, se logra desarrollar la investigación resaltando características importantes de los ingresos de las personas, la falta de políticas salariales en las instituciones públicas y privadas. También hacemos un llamado a la existencia de colegios profesionales con poca 0 ninguna relevancia en el mejoramiento de la remuneración salarial de los empleados o aportantes, tanto de las empresas privadas como públicas.

Esto conduce a mostrar la posible existencia de un punto ciego en la remuneración salarial. Desde el inicio se conoce la existencia de un punto de partida en la remuneración salarial, como el salario mínimo, y de aquí en adelante la remuneración salarial la determina tanto la oferta como la demanda en el mercado laboral y uno que otro colegio profesional, lo que implica que individuos con un nivel de educación superior tienen que aceptar que el empleador les pague una remuneración baja y no la que se podrían merecer como ofertantes de mano de obra más calificada. 


\section{Especificación teórica del modelo}

La teoría del capital humano constituye una de las más importantes contribuciones para el estudio de la economía de la educación, dado que representa un marco importantísimo para la comprensión racional de la inversión en educación y formación y para la apreciación de la rentabilidad económica y social de esa inversión.

Mincer (1958) presenta un modelo para tratar de determinar cuánto debe educarse una persona si es que quiere maximizar su riqueza inter temporal (no utilidad). Supone que todos los individuos tienen las mismas habilidades y oportunidades de entrar a una ocupación, pero estos trabajos difieren en la cantidad de entrenamiento que requieren. Educarse tiene costos indirectos, como los salarios perdidos durante el periodo educativo y la reducción del periodo para recuperar la inversión.

Para esta investigación la función base será la que expresan en sus documentos (Merlo Juan, 2009) "Retornos a la educación durante una depresión económica" y (Freire Seoane, María de Jesús 2008) "Las ecuaciones de Mincer y las tasas de rendimiento de la educación en Galicia", quienes se basan en la idea originaria de la ecuación minceriana. Esta la construyen sobre identidades contables y es el estudio clásico que postula una relación positiva entre el logaritmo natural (Ln) de los ingresos con los años de educación y experiencia. Se focaliza en la dinámica de los ingresos a lo largo del ciclo de vida y con la relación entre ingresos observados, ingresos potenciales e inversión en capital humano, tanto en términos de educación formal como de inversión en el trabajo, la función es la siguiente:

$$
\operatorname{Ln}(Y)=\beta 0+\beta 1 S+\beta 2 \operatorname{Exp}+\beta 3 \operatorname{Exp} 2+\varepsilon \quad \text { (1) (Freire, 2008) }
$$

Esta función expresa el logaritmo de los ingresos ( $\operatorname{Ln} Y$ ) igualado a los años de educación (S), experiencia (Exp) y la experiencia al cuadrado (Exp2). De esta manera, llega a resultados bastante coherentes, en distintos países y en diversos períodos de tiempo, predice bastante bien la distribución del ingreso, aunque no haya sido confeccionado para ello.

Uno de los problemas que menciona la teoría, con relación al modelo lineal, es que la función pretende resultados idénticos o únicos independientemente de los distintos niveles de escolaridad que el individuo alcanza. Visto de otra manera, un modelo de regresión lineal presenta heterocedasticidad cuando la varianza no es constante a lo largo de las observaciones, esto significa el incumplimiento de una de las hipótesis básicas sobre las que se asientan estos modelos lineales. Sin embargo, se deriva 
que los datos con los que se trabaja son heterogéneos, con distinta varianza.

Existen diferentes razones o situaciones en las que cabe encontrarse con perturbaciones heteroscedásticas. La situación más frecuente es en el análisis de datos de corte transversal, ya que los individuos, empresas o unidades económicas no suelen tener un comportamiento homogéneo. Sin embargo, cuando se realicen las estimaciones econométricas se realizarán las pruebas para poder reparar las regresiones por heterocedasticidad,

Detalles de pruebas estadísticas de heterocedasticidad

El modelo básico de regresión lineal, según Rafael de Arce y Mahia Ramon, en el documento Conceptos básicos sobre la Heterosedasticidad en el Modelo Básico de Regresión Lineal, exige como hipótesis básica que la varianza de las perturbaciones aleatorias, condicional a los valores de los regresores $\mathrm{X}$, sea constante:

\section{Utilizar mínimos cuadrados ordinarios (MCO) ignorando la heterocedasticidad}

En este caso, tenemos una varianza que, dado el error de cálculo antes mencionado, es un estimador sesgado del verdadero valor de la varianza (valor correctamente calculado) sin que, en general, se pueda saber si ese cálculo incorrecto sobreestima o subestima el verdadero valor. Conviene, además, tener en cuenta que el problema del cálculo incorrecto deriva en realidad de que el estimador insesgado de la varianza de la perturbación ya no resulta ser matemáticamente asi:

$$
\frac{\sigma^{2}=e^{\prime} e}{n-k}
$$

De modo que, además del error de cálculo en la estimación de la varianza de los parámetros, todos aquellos contrastes o test basados en este estimador insesgado serán también incorrectos. En todo caso, un error frecuente consiste en pensar que cualquier cálculo que implica la utilización de los errores de un modelo heterocedastico; será incorrecto cuando, en realidad, no es así, según (De Arce, Rafael y Mahia, Ramon, 2009).

Un ejemplo interesante es el cálculo del coeficiente de determinación R2 (o su versión corregida) que no se ve afectado por la existencia de heterocedasticidad. La razón estriba en que el cálculo de la $\mathrm{R} 2$ se realiza a partir del cálculo de las varianzas poblaciones de "u" ( $\sigma u)$ y de "y" ( $\sigma y)$ y el hecho de que se usen conceptos, poblaciones, no muestrales; implica que utilizan varianzas no condicionales a los valores de 
"x", de modo que la R2 (poblacional) no se ve afectada por la presencia de heterocedasticidad; de hecho, la expresión arriba escrita estima consistentemente la varianza

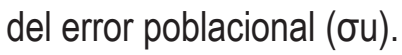

\section{Utilizar mínimos cuadrados generalizados (MCG)}

Al utilizar los mínimos cuadrados generalizados (MCG) como una de las mejores soluciones, también deben de observarse que la utilización de este estimador exigiría conocer o estimar de antemano los valores de los elementos del conjunto de sigmas $(\Sigma)$. Estimar las " $n$ " varianzas distintas del conjunto de sigmas $\Sigma$ partiendo de " $n$ " observaciones y "k" variables explicativas es imposible, de modo que, como se verá más adelante, la utilización de este estimador exigirá asumir algún supuesto simplificador sobre la causa de una eventual heterocedasticidad, este permite a su vez determinar, de forma también simplificada, la forma de la matriz de los conjuntos de sigma. Evidentemente, encontrar una simplificación correcta de este conjunto de sigmas dotará de plena utilidad (eficiencia) a la estimación con MCG; de lo contrario, un mal diseño de la causa de la heterocedasticidad y su expresión en el conjunto de sigmas no garantizará esa eficiencia.

Cómo detectar la presencia de heterocedasticidad

Antes de entrar a enumerar y revisar brevemente los principales procedimientos, deben quedar claras dos cuestiones preliminares:

1. Resultará imposible observar directamente la presencia de heterocedasticidad, ya que en la mayoría de los análisis econométricos solo se disponen de un valor de " $\mathrm{y}$ " para cada valor de "x" (por tanto de un único valor de "u"); por lo que resulta conceptualmente imposible observar si la varianza de las " $u$ " para cada valor de " $x$ " es la misma. Por tanto, la mayor parte de los métodos se apoyarán en los residuos obtenidos en un modelo previo (estimado generalmente con MCO); estos residuos se utilizarán como una muestra válida de las perturbaciones aleatorias desconocidas.

2. Antes de la aplicación de métodos técnicos (más o menos informales) hay que preguntarse por la existencia de heterocedasticidad desde un punto de vista teóri$\mathrm{co}$, considerando la naturaleza del problema analizado, las exógenas incluidas y, en definitiva, la propensión teórica del modelo hacia la heterocedasticidad.

Según Rafael de Arce y Mahia Ramon, en el documento Conceptos básicos sobre la 
Heterosedasticidad en el Modelo Básico de Regresión Lineal, plantean como una breve receta, los pasos para la corrección de la heterocedasticidad serían:

1. Se estiman los parámetros del modelo por MCO, ignorando por el momento el problema de la heterocedasticidad de las perturbaciones aleatorias.

2. Se establece un supuesto acerca de la formación de las varianzas $\left(\sigma^{2}\right)$ y se emplean los residuos de la regresión por MCO para estimar la forma funcional supuesta.

3. Se divide cada observación por $\left(\sigma^{2}\right)$ según el paso anterior (según el valor de esa heterocedasticidad supuesta estimada, siempre que un contraste haya confirmado que el modelo simplificador es bueno).

4. Se estima el modelo original con todas las variables transformadas según el paso 3.

Partiendo de las especificaciones que realiza Friere en su documento "Las ecuaciones de Mincer y las tasas de rendimiento de la educación en Galicia", (Barcenias, Fernando 1999), usa como base la función No. 1 siguiente:

$$
\operatorname{Ln}(Y)=\beta 0+\beta 1 S+\beta 2 \operatorname{Exp}+\beta 3 \operatorname{Exp} 2+\varepsilon(1)
$$

En la cual modela la existencia de $m$ niveles de escolaridad, en esta asocia cada nivel $\mathrm{dk}$, como los distintos niveles educativos, utiliza $\mathrm{K}=$ sin educación (0), educación primaria (1), educación secundaria (2), y educación superior (3); de esta manera, siguiendo las especificaciones de Psacharopoulos, se obtienen las tasas de rentabilidad para cada nivel educativo comparando los coeficientes de dk con dk-1 y los divide por nk, o sea, por el número de años escolares correspondientes a cada nivel educativo. La metodología de Barcenias se plantea de la siguiente ecuación:

$$
\frac{\mathrm{Dn}-\mathrm{Dn}-1}{\mathrm{~N}}
$$

Dn: es el nivel de educación a calcular la tasa de retorno.

Dn-1: es el nivel de educación anterior a calcular.

$\mathrm{N}$ : los años de estudio promedios para culminar determinado grado.

Se reconoce que el proceso de educación es acumulativo y, por consiguiente, se debe tener un valor neto para cada año de estudio aumentado. 


\section{METODOLOGÍA}

\section{Enfoque de la investigación}

El tipo de investigación a desarrollarse es mixto, explicativo, no experimental, de corte trasversal.

El tipo de investigación a desarrollarse tendrá dos etapas: en primer lugar, en la parte cuantitativa se implementará un modelo econométrico con el fin de aplicar las bases teóricas que respaldan el modelo experimental. La segunda etapa corresponde a un enfoque cualitativo, basado en información que se recolecte de determinada fuente primaria. Para esta etapa la investigación tomará como base las variables que están dentro del rango según los objetivos propuestos, tales como la experiencia, años de estudios, los niveles de ingreso y sexo, detallados en la operacionalización de variables.

De esta manera, se podrá tener un panorama muy sólido que permitirá que los objetivos propuestos sean perfectamente evidenciados al final de la investigación. También se podrá realizar un análisis sobre los resultados que se realicen con ambos enfoques.

\section{Diseño de investigación}

Teniendo en cuenta que la investigación comprende dos etapas, el diseño que se desarrolló es de tipo explicativo, no experimental, de corte trasversal. Así, especificamos la estructura de cómo se dirigirá este proyecto de investigación por medio del cual tratamos de cumplir con los objetivos de la investigación. Para cumplir con los objetivos planteamos los siguientes pasos:

a. Temporalidad de la investigación.

b. Determinar la población y muestra.

c. Hipótesis de investigación.

d. Definición de la información.

\section{Temporalidad de la investigación}

La temporalidad de esta investigación está fundamenta según el tipo de modelo econométrico que se usa y la disponibilidad de la información, ya sea un modelo de 
serie de tiempos (que existan variables con información estadística de varios años) 0 en este caso de corte trasversal (que determina la existencia de información estadística en un punto de la historia).

\section{Población y muestra}

La primera etapa, la muestra, es no probabilística obtenida por conveniencia, dadas las circunstancias del uso de la base de datos y la relevancia del análisis. Primero se tendrá que limpiar la base de datos por medio de sesgo de selección, para compactarla usando las variables determinantes para poder implementar el modelo econométrico.

Para el análisis cualitativo se tomará como base a las personas que tuvieron trabajo en los últimos 3 meses anteriores y que estudian en la Universidad Nacional Autónoma de Honduras. Tomando en cuenta que la mayoría de personas que trabajan y estudian se encuentran en la jornada nocturna, se obtiene el universo que será estratificado por facultades; de esta manera se puede llegar hasta las carreras; asimismo, tener una muestra representativa de todas las carreras. Luego, a través de un muestreo aleatorio simple, llegar a las unidades de análisis, en este caso los estudiantes, y así determinar una muestra por carreras.

\section{Cuadro 1. Muestra representativa de estudiantes de la ciudad universitaria}

\begin{tabular}{|l|l|l|}
\hline No. & Estratos/ Facultades & ni \\
\hline 1 & Humanidades & 18 \\
\hline 2 & Ciencias sociales & 15 \\
\hline 3 & Ciencias & 10 \\
\hline 4 & $\begin{array}{l}\text { Ciencias agrícolas y } \\
\text { ambientales }\end{array}$ & 0 \\
\hline 5 & Ciencias económicas & 36 \\
\hline 6 & Ciencias jurídicas & 7 \\
\hline 7 & Ciencias médicas & 19 \\
\hline 8 & Química y farmacia & 7 \\
\hline 9 & Ingeniería & 17 \\
\hline 10 & Odontología & 8 \\
\hline 11 & Ciencias espaciales & 0 \\
\hline & Total & 138 \\
\hline
\end{tabular}

Fuente: Elaboración propia con datos de la Secretaria Ejecutiva de Desarrollo Institucional de la UNAH, año 2014. 


\section{Cuadro 2. Operacionalización de variables}

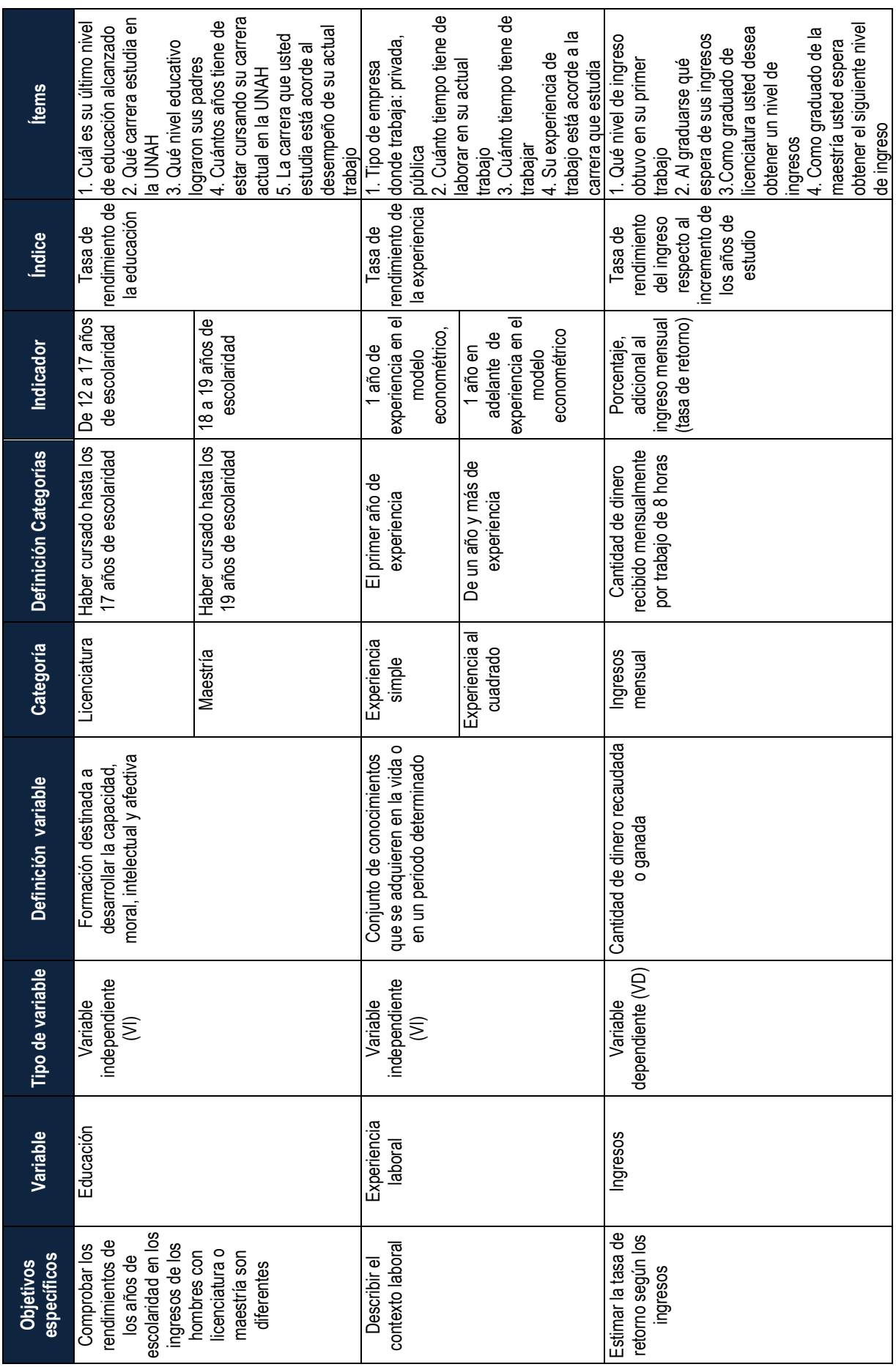

Fuente: elaboración propia basada en información de la EPHPM, 2012. 


\section{Cuadro 3. Operacionalización de categorías}

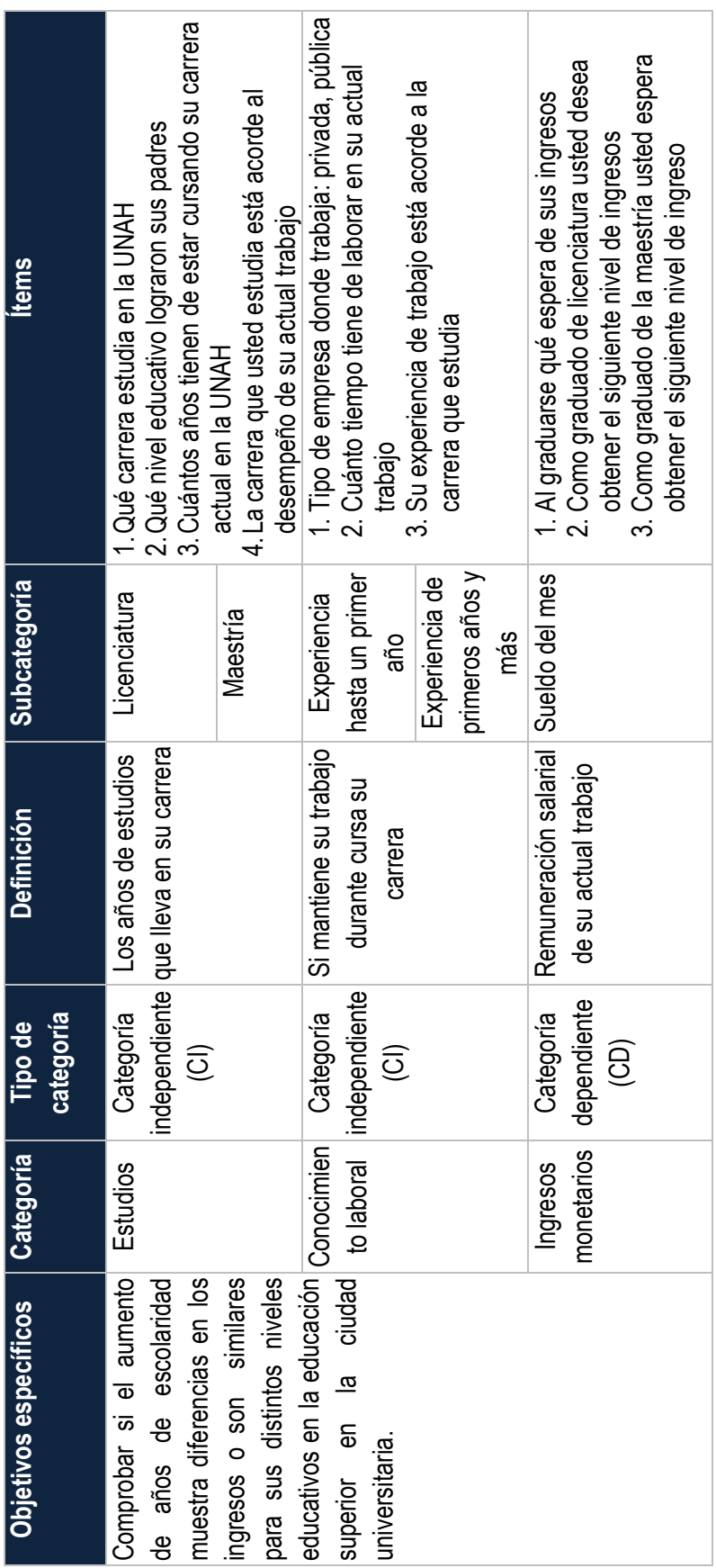

Fuente: elaboración propia basados en información de la EPHPM, 2012. 
La operacionalización de variables se desarrolla como base en el enfoque del estudio y el marco teórico, permitiendo llegar a las variables descritas realizando el análisis correspondiente.

\section{RESULTADOS}

A nivel nacional se determinaron que el nivel de pobreza relativa es del $23.2 \%$ y el de pobreza extrema es del $42.7 \%$; en consecuencia, los no pobres se estiman en un $34.1 \%$, a través de este método se cuantifica la pobreza total en un $65.9 \%$ y esto guarda relación con las estimaciones realizadas de manera oficial por el foro social de la deuda externa. (FOSDEH, 2014).

Naturalmente, el método del ingreso se puede analizar a la luz de la influencia de la educación en la pobreza medida a través del ingreso. En este caso particular de la educación, se basa en el nivel educacional alcanzado por el jefe de hogar, esto con respecto a la medición de la pobreza a través del ingreso, el cual debiera revelarse como una relación más directa en el caso que se tomaran el indicador de Necesidades Básicas Insatisfechas (NBI), ya que es de esperar que conforme avanza el nivel de escolaridad en similar medida disminuye la proporción de pobreza y extrema pobreza, por estar asociada a la mayor percepción de ingresos de los hogares con mayor escolaridad.

Según cifras que reporta el Banco Central de Honduras para el año 2014, en cuanto al nivel de escolaridad alcanzado por los migrantes antes de salir del país, el $97.0 \%$ tuvo alguna formación académica; destacándose los que completaron la educación primaria con $33.0 \%$, seguidos por los que culminaron la secundaria o educación media (28.0\%). Sin embargo, el $48.9 \%$ de los encuestados aseguró no haber cursado estudios académicos en el extranjero. El $20.8 \%$ decidió enfocar sus esfuerzos en el aprendizaje del idioma (principalmente el inglés) del país de residencia, mientras que el $12.9 \%$ de los entrevistados completó una carrera universitaria o especialización de posgrado. Asimismo, $12.5 \%$ se especializaron como técnicos en mecánica, enfermería y computación, entre otros.

El acercamiento econométrico que se desarrolla es un modelo probabilístico que tiene como variable dependiente el ingreso en función de los años de escolaridad y la experiencia basada en la encuesta de hogares del INE de 2013. El marco muestral, tal como se menciona en la metodología, se reduce a la población entre 15 y 64 años 
de edad y a los diferentes niveles educativos correspondientes.

Para la variable dependiente se utilizó el ingreso per cápita por hogares usada por la base de datos. En donde los ingresos más altos están dados por L.7, 682.16; lo que corresponde al quintil 5 y el más bajo está dado por L. 379.66 en el quintil 1; lo que está relacionado con los niveles de escolaridad, reflejan que la población del primer quintil tiene en promedio 5.7 años de escolaridad con respecto a los del quintil 5 que doblan el promedio, obteniendo 10.9 años de escolaridad.

La anterior descripción da como resultado un modelo econométrico que se muestra a continuación:

1. Logit Yquintil: es el logaritmo del ingreso de los hogares por quintil.

2. Exp: representa la experiencia el primer año.

3. Exp2: representa la experiencia de 2 años o más.

4. i.Nivel: los diferentes niveles educativos que van desde sin nivel educativo, educación primaria, educación secundaria y educación superior.

La experiencia tanto en el primer año como en los demás, no es significativa para el aumento de los niveles de ingresos de la población, generando una probabilidad de que el ingreso disminuya en un $7 \%$; contrario a la probabilidad de los niveles educativos.

El nivel educativo 3 representa los años de escolaridad agrupados hasta el nivel de educación secundaria con una probabilidad de aumento del $23.2 \%$, un valor que se mira en aumento a medida que los años de escolaridad crezcan y llegan hasta los años de educación superior (nivel educativo 4) que tienen una probabilidad promedio de un $45 \%$. Ambos coeficientes son significativos técnicamente y se apegan a la teoría respaldándola y aumentando la credibilidad en seguir escalando los diferentes niveles educativos para tener como recompensa mejores salarios.

Tal como lo menciona la investigación sobre el análisis de la pobreza en Honduras, (FOSDEH, 2014) la educación es una de las columnas para el desarrollo y salir de la pobreza y extrema pobreza en el área urbana y rural. Cabe mencionar que la variable experiencia, aunque no es significativa, no se debe dejar de lado, porque el hecho empírico que disminuya el nivel de ingreso determina que para el mercado laboral de nuestro país se prefiere mejor a alguien que tenga un título a alguien que tenga años de experiencia, solo con el fin, probablemente, de pagar determinado salario y de esta manera disminuir costos operativos en sus empresas. 


\section{Resultados del acercamiento empírico en la ciudad universitaria}

Según la información de obtenida en la Ciudad Universitaria, entre los meses de septiembre y octubre del 2014, podemos mencionar que los ingresos de la población varían según el tipo de empresa, la composición de la población representada pertenecen a la empresa privada en un $68.1 \%$; en un segundo lugar la empresa pública con un $19.3 \%$. Otra parte toma rumbos diferentes, tales como las ONG, con un 4.4, lo mismo para los que laboran por cuenta propia.

Al realizar cruces de variables se observa que alrededor del $15 \%$ de la población que tiene menos de un año de trabajar ha laborado en la empresa pública y en la empresa privada. Cabe mencionar que los ciclos de cuatro años de gobierno se muestran con mayor claridad, ya que no todos los empleados logran agenciarse de un puesto de trabajo en sus siguientes tres años, es por eso que la población que trabaja en la empresa pública los siguientes tres años disminuye a un porcentaje del $4 \%$, en cambio cuando tienen más de tres años de experiencia laboral la población aumenta a un $46 \%$.

Caso contrario sucede en las empresas privadas, en donde inician con un $15 \%$ y aumentan en su primer año a un $22 \%$, con una disminución de 7 puntos porcentuales con respecto al tercer año, pero los empleados que mantienen su trabajo en la empresa privada y tienen más tres años de experiencia llegan a un $36 \%$.

Tanto las ONG y los trabajadores por cuenta propia que tienen experiencia en los primeros años son muy similares en sus movimientos laborales y al final después de los tres años de experiencia se mantienen.

\section{CONCLUSIONES}

Al analizar el aumento de los años de escolaridad y su relación sobre los ingresos mensuales, se obtuvo que el impacto es positivo, observando que al incrementar la variable niveles de educación, los nivel de ingresos aumentan; es así como los resultados del modelo probabilístico desarrollado muestra que los niveles de educación según los años de escolaridad agrupados para los niveles de educación secundaria y educación superior, obtuvieron resultados como ser nivel secundaria una probabilidad de que el ingreso aumente de un $23.2 \%$, mientras que la educación superior tiene una probabilidad del $45 \%$. 
Cabe mencionar que a nivel nacional, según al Análisis de la Pobreza en Honduras del FOSDEH el nivel de pobreza relativa es del $23.2 \%$ y de la pobreza extrema es del $42.7 \%$; de esta manera, se cuantifica la pobreza total en un $65.9 \%$. Sin embargo, en el otro extremo del nivel educacional, a mayor escolaridad, se observan mayores proporciones de hogares no pobres; es muy contundente la evidencia de que el ingreso aumenta conforme avanzan los años de escolaridad.

Al estimar la tasa de retorno para el área de Tegucigalpa, según los ingresos dio como resultado una tasa de retorno del $23 \%$ y un $45 \%$ para la educación secundaria y educación superior.

Al evaluar los objetivos propuestos se comprueba que los años de escolaridad tuvieron mayor impacto en los ingresos de los individuos que tienen licenciatura, muy diferente en el caso de maestría; al comparar ambos niveles se determinó que existe diferencia en el caso de los ingresos para profesionales con maestría, pues sus ingresos se inician a percibir entre el valor de L. 13,001.00 y 16,000.00; mientras que los niveles salariales de las personas que tienen el nivel de licenciatura es similar y existe mayor población que tiene ese mismo nivel de ingreso.

También se comprobó que los ingresos en la ciudad universitaria difieren por el nivel educativo, los profesionales en el nivel de secundaria inician un repunte con un $28 \%$ de representatividad de la población universitaria; diferente para los que tienen el nivel de licenciatura, que solo representa el $4 \%$ de la población que obtiene estos ingresos. Esto resulta interesante porque un egresado del nivel de licenciatura obtiene un ingreso muy bajo.

Existe más variación en el tercer rango de L. 10,000.00 a 13,000.00; en donde disminuye en 15 puntos porcentuales la población de secundaria que obtiene este ingreso. Para el cuarto nivel la población que alcanza este ingreso son muy similares, con la diferencia de un punto porcentual para la población del nivel de licenciatura.

Sin duda se comprobó que los ingresos varían de acuerdo a los años de estudios de las personas, los egresados de la educación secundaria obtienen un ingreso de menos de L. 8,000.00 hasta los 19,000.00; el nivel de licenciatura obtiene casi lo mismo, con la diferencia de que han obtenido más de L. 19,000.00; lo que muestra una diferencia significativa con relación a que es más rentable seguir estudiando. Sin embargo, entre el nivel de licenciatura y maestría, se encontró en este último que el inicio del ingreso para este nivel es de L. 13,001.00 hasta obtener más de L. 19,000.00; similar al nivel de licenciatura. De esta manera, es posible mencionar que 
la hipótesis 1 no se cumple, de modo que los ingresos de la población que tiene nivel de maestría es similar o mayor, pero no menor.

La hipótesis sobre el nivel de ingreso de los jóvenes que alcanzan el nivel de licenciatura obtienen los L. $16,000.00$ y 19,000.00; solamente un $6 \%$ en contraste con la población que tiene maestría, que representa un $33 \%$ y de L. 19,000.00 en adelante los jóvenes con licenciatura representan el $11 \%$ y con maestría un $67 \%$; una diferencia muy considerable. De esta manera se cumple la hipótesis de que los jóvenes con licenciatura obtienen menor ingreso que los que tienen maestría.

\section{BIBLIOGRAFÍA:}

Barceinas, Fernando. (1999). Los rendimientos de la educación en España. Clasificación JEL:I21, España-Editorial Comisión Europea en su proyecto: PURE SOE2-CT98-2044 "Public funding and private returns to educations" asi como a CCYT SS 97-1333 y a la fundación FUNCAS

$\mathrm{BCH}$. (2014). Remesas familiares. Tegucigalpa- BCH

De Arce Rafael y Mahia Ramón, (2009) Conceptos básicos sobre la Heterosedasticidad en el Modelo Básico de Regresión Lineal. Universidad Autónoma de Madrid/ Depto. de Economía Aplicada.

FOSDEH. (2013-2014). Análisis de la pobreza en Honduras. Caracterización y análisis de determinantes. Tegucigalpa, Honduras: publicada por FOSDEH.

Freire Seoane, María de Jesús. (2008). Las ecuaciones de Mincer y las tasas de rendimiento de la educación en Galicia. Universidad de A. Coruña, España, Capitulo 14 de Investigación de Economía de la Educación, 2010, Vol. 5, pp 285-304 de la Asociación de la Economía de la educación.

INE, (2014) Encuesta Permanentes de Hogares para Usos Múltiples.

Merlo, Juan. (2009). Retornos a la educación durante una depresión económica. Evidencia empírica para la Argentina. LUGAR: Pontificia Universidad Católica de Chile.

PNUD. (2011). Informe de Desarrollo Humano Honduras 2011. Reducir la inequidad: un desafío impostergable. LUGAR.

Secretaría de Educación. (1997). Estudio Sectorial-Plan Decenal. Tegucigalpa. 